\title{
IMPROVED SOBOLEV INEQUALITIES
}

\author{
BY
}

\author{
ROBERT S. STRICHARTZ
}

\begin{abstract}
For a function $f$ defined on $\mathbf{R}^{n}$. Sobolev's inequality $\|f\|_{q} \leqslant$ $c\left(\|f\|_{p}+\|\nabla f\|_{p}\right)$, where $1<p<q<\infty$ and $1 / p-1 / q=1 / n$, can be improved if the Fourier transform $\hat{f}$ is assumed to have support in a set $A$ which satisfies an estimate $|\{\xi \in A:\|\xi\| \leqslant s\}| \leqslant c s^{d}$ for some $d<n$; the improvement being that we can take $1 / p-1 / q=1 / d$, provided we also assume $p \leqslant 2 \leqslant q$. Analogous results are proved for other Sobolev inequalities, for embeddings into Lipschitz-Zygmund spaces, and for functions on symmetric spaces whose Fourier expansions are suitably limited. Improved Sobolev inequalities are established locally for solutions of the wave equation. An application to the Radon transform on spheres is given.
\end{abstract}

1. Introduction. Consider the Sobolev inequality $\|f\|_{q} \leqslant c\left(\|f\|_{p}+\|\nabla f\|_{p}\right)$ for $f$ defined on $\mathbf{R}^{n}$, where $1 \leqslant p<q<\infty$ and $1 / p-1 / q=1 / n$. Simple examples show that it cannot be improved, in the sense that the index $q$ cannot be increased. However, if we are willing to restrict the class of functions $f$ in some way, then the possibility arises of improving Sobolev's inequality. One trivial way to do this is to assume that $f$ is only a function of $d$ variables, for $d<n$. Then, at least locally, we can take $1 / p-1 / q=1 / d$. In this paper we consider other, less trivial, ways to restrict $f$ to obtain the same sort of improvement.

In $\$ 2$ we consider functions whose Fourier transforms are supported in a set $A$ which satisfies an estimate $|\{\xi \in A:|\xi| \leqslant s\}| \leqslant c s^{d}$ for some $d<n$ (not necessarily an integer). We will say that $A$ has asymptotic dimensions $\leqslant d$. We will show that the Sobolev inequality holds for $1 / p-1 / q=1 / d$ for such $f$, provided $p \leqslant 2 \leqslant q$. This condition is essential for our method, which is nothing other than a trivial modification of an old proof of the usual Sobolev inequality due to Hardy, Littlewood and Paley. It can be relaxed if the characteristic function of $A$ is an $L^{p}$ Fourier multiplier for $1<p<\infty$, but not in general, as is shown by a counterexample in $\S 5$.

$\S 2$ also contains a sampling of related improved Sobolev inequalities (with $d$ in place of $n$ ) involving Lipschitz-Zygmund classes when $p>d$ and exponential integrability when $p=d$. We actually obtain all results for functions in Sobolev spaces $L_{\alpha}^{p}$ of all orders, not just $\alpha=1$.

We consider analogous results for functions on compact Lie groups or compact symmetric spaces in $\S 3$. Here there is a natural Fourier series expansion $f=\Sigma_{m \in \mathfrak{L}} f_{m}$, and we consider functions whose expansion is limited to $f_{m}$ with $m \in A$. Many of the

Received by the editors December 14, 1981 and, in revised form, November 12, 1982.

1980 Mathematics Subject Classification. Primary 46E35, 42B99; Secondary 43A77, 43A85, 35L05.

Key words and phrases. Sobolev inequality, symmetric space, Radon transform, wave equation.

'Research supported in part by NSF Grant MCS-8002771. 
proofs are similar to those of $\S 2$, so we only give sketches of the proofs. These results are applied in $\S 4$ to the Radon transform on spheres. We are able to improve estimates in [14] that were obtained by applying the usual Sobolev inequalities to functions on the Grassmannian manifold $O(n+1) / O(n-k) \times O(k+1)$ whose Fourier series expansions are limited to spherical harmonic representations. This was actually the problem that originally motivated this paper, providing the first clue that improved Sobolev inequalities might be true and interesting.

In $\$ 5$ we derive local improved Sobolev inequalities for solutions of the wave equation. Here the situation is more complicated, in that the difference $1 / p-1 / q$ is not constant, but varies between the expected value and a higher value as $p$ varies. We are also able to construct simple counterexamples that shed light on the restriction $p \leqslant 2 \leqslant q$ in $\S 2$.

Finally in $\S 6$ we discuss analogous results on noncompact symmetric spaces.

On a technical level this paper is extremely simple. All the proofs are straightforward extensions of known results. Therefore we have tried to keep the presentations as brief as possible. The reader is referred to [7,8,15, 16, and 17] for background material.

We are grateful to J. Gilbert and D. Ragozin for helpful comments.

2. Euclidean space. Let $A \subseteq \mathbf{R}^{n}$ be a measurable set of nonzero measure. If the inequality

$$
|\{\xi \in A:|\xi| \leqslant s\}| \leqslant c s^{d}
$$

holds for some constant $c$ and all $s>0$, we will say that $A$ has asymptotic dimension $\leqslant d$. We will only be interested in the case $d<n$ since (2.1) always holds for $d=n$. Note that (2.1) is then automatic for $s<1$. Let $P_{A}$ denote the orthogonal Fourier projection onto $A, P_{A} f=\mathscr{F}^{-1}\left(\chi_{A} \hat{f}\right)$, where $\chi_{A}$ denotes the characteristic function of $A$. Let $L_{\alpha}^{p}$ denote the Sobolev space of Bessel potentials of order $\alpha$ of $L^{p}$ functions, $1<p<\infty$ (see [7]).

THEOREM 2.1. Fix A of asymptotic dimension $\leqslant d$. Then

$$
\left\|P_{A} f\right\|_{L_{\beta}^{q}} \leqslant c\|f\|_{L_{\alpha}^{p}} \text { for all } f \in L_{\alpha}^{p}
$$

provided

$$
1<p \leqslant 2 \leqslant q<\infty \text { and } 1 / p-1 / q=(\alpha-\beta) / d .
$$

In particular, the improved Sobolev inequality

$$
\|f\|_{L_{\beta}^{q}} \leqslant c\|f\|_{L_{\alpha}^{p}}
$$

holds if $\hat{f}$ has support in $A$ and (2.3) holds.

Proof. It suffices to show $J_{\alpha-\beta} P_{A}$ is a bounded operator from $L^{p}$ to $L^{q}$, where $J_{\alpha-\beta}$ denotes the Bessel potential operator. This is a simple consequence of a theorem of Paley, whose proof we review briefly. A well-known theorem of Hardy and Littlewood, whose proof is now a simple consequence of the interpolation theory of Lorentz spaces [8], gives the estimates

$$
\|\hat{f}\|_{L^{p^{\prime}, p}} \leqslant c\|f\|_{L^{p}} \text { if } 1<p \leqslant 2
$$


and

$$
\|f\|_{L^{4}} \leqslant c\|\hat{f}\|_{L^{4^{\prime} \cdot 4}} \text { if } 2 \leqslant q<\infty .
$$

Here $L^{p, q}$ denotes the Lorentz space. If $T_{m} f=\mathscr{F}^{-1}(m \hat{f})$ is any Fourier multiplier operator with $m \in L^{r, \infty}$ with $p^{-1}-q^{-1}=r^{-1}$, then Paley's theorem asserts that $T_{m}$ is bounded from $L^{p}$ to $L^{q}$ (with $1<p \leqslant 2<\mathrm{q}<\infty$ ), and this follows immediately from (2.5), (2.6) and the fact that multiplication by $m$ is a bounded operator from $L^{p^{\prime} \cdot p}$ to $L^{q^{\prime} \cdot q}$ (this can be proved easily by interpolation since $p \leqslant q$ ).

Now the operator $J_{\alpha-\beta} P_{A}$ is a Fourier multiplier operator with

$$
m(\xi)=\left(1+|\xi|^{2}\right)^{(\beta-\alpha) / 2} \chi_{A}(\xi),
$$

and it is an easy consequence of (2.1) that $m \in L^{r . \infty}$ with $r^{-1}=(\alpha-\beta) / d$. Q.E.D.

RemarKs. (1) An alternate proof involves first assuming $(\alpha-\beta) / d>1 / 2$, so that $r<2$. Then by a generalization of (2.6), $J_{\beta-\alpha} P_{A}$ is convolution with a function in $L^{r^{\prime}, \infty}$, which is bounded from $L^{p}$ to $L^{q}$. To remove the restriction on $\alpha-\beta$ we use an analytic families interpolation argument (as in the proof of Theorem 2.2 below). While this alternative seems unnecessarily complicated in this context, we will need to use analogous reasoning in other contexts.

(2) It seems tempting to try to interpolate between (2.4) and the usual Sobolev inequalities to obtain weaker improved estimates without the assumption $p \leqslant 2 \leqslant q$. However, this does not work, because the support of $\hat{f}$ cannot be kept in $A$ while varying $p$. Cf. the counterexample in $\S 5$.

THEOREM 2.2. Fix $A$ of asymptotic dimension $\leqslant d$ and assume, in addition, that $P_{A}$ is bounded on $L^{p}$ for $1<p<\infty$. Then the hypothesis $p \leqslant 2 \leqslant q$ may be omitted in Theorem 2.1. If we only assume that $P_{A}$ is bounded on $L^{p}$ for $p_{0}<p<p_{0}^{\prime}$, then Theorem 2.1 remains true for $1<p<q<2$ if $\left(\frac{1}{p}, \frac{1}{q}\right)$ lies in the interior of the triangle with vertices $\left(\frac{1}{2}, \frac{1}{2}\right),\left(1, \frac{1}{2}\right),\left(\frac{1}{p_{0}}, \frac{1}{p_{0}}\right)$, and similarly for $2<p<q<\infty$ if $\left(\frac{1}{p}, \frac{1}{q}\right)$ lies in the interior of the triangle with vertices $\left(\frac{1}{2}, \frac{1}{2}\right),\left(\frac{1}{2}, 0\right),\left(\frac{1}{p_{0}^{\prime}}, \frac{1}{p_{0}^{\prime}}\right)$.

Proof. Consider the analytic family of operators $J_{z} P_{A}$ in $0 \leqslant \operatorname{Re} z \leqslant d / 2$. On the line $\operatorname{Re} z=0$ we have $L^{p}$ boundedness for $p_{0}<p<p_{0}^{\prime}$, since we have assumed it for $P_{A}$ and $J_{z}$ is known to be bounded on $L^{p}$ for $1<p<\infty$ (see [7]). On the line $\operatorname{Re} z=d / 2$ we have boundedness from $L^{p}$ to $L^{q}$ if $1 / p-1 / q=1 / 2$ by Theorem 2.1. The result follows by Stein's interpolation theorem for analytic families [8]. Q.E.D.

Next we consider analogous results involving Lipschitz spaces. Let $\Lambda_{\alpha}$ denote the Lipschitz-Zygmund class of all bounded functions (when $\alpha>0$ ) satisfying

$$
\left|\left(\frac{\partial}{\partial x}\right)^{\beta} f(x+y)+\left(\frac{\partial}{\partial x}\right)^{\beta} f(x-y)-2\left(\frac{\partial}{\partial x}\right)^{\beta} f(x)\right| \leqslant M|y|^{\alpha-k}
$$

for all $|\beta|=k$ where $0<\alpha-k<2$. For our purposes we need a characterization of $\Lambda_{\alpha}$ in terms of a Littlewood-Paley dyadic annuli decomposition of $f$. Let $\sigma, \tau \in \mathcal{S}$ $\left(\mathbf{R}^{n}\right)$ be chosen so that $\hat{\sigma}$ is identically one on the annulus $1 \leqslant|\xi| \leqslant 2$ and vanishes outside the larger annulus $1 / 2 \leqslant|\xi| \leqslant 4$, while $\hat{\tau}$ is identically one on the ball 
$|\xi| \leqslant 1$ and vanishes outside the ball $|\xi| \leqslant 2$. Let $\sigma_{k}(x)=2^{n k} \sigma\left(2^{k} x\right)$, so $\hat{\sigma}_{k}(\xi)=$ $\hat{\sigma}\left(2^{-k} \xi\right)$ is identically one on the annulus $2^{k} \leqslant|\xi| \leqslant 2^{k+1}$ and is supported in the larger annulus $2^{k-1} \leqslant|\xi| \leqslant 2^{k+2}$. Then for $\alpha>0$,

$$
f \in \Lambda_{\alpha} \Leftrightarrow\|\tau * f\|_{\infty} \leqslant M \text { and }\left\|\sigma_{4} * f\right\|_{\infty} \leqslant 2^{-k \alpha} M
$$

for all nonnegative integers $k$ (see [2]). The least constant $M$ in (2.7) is equivalent to the $\Lambda_{\alpha}$ norm of $f$.

Theorem 2.3. Fix $A$ of asymptotic dimension $\leqslant d$. Suppose $\alpha>d / p, 1<p \leqslant 2$, and $\beta=\alpha-d / p$. Then

$$
\left\|P_{A} f\right\|_{\Lambda_{\beta}} \leqslant c\|f\|_{L_{-a}^{p}} \text { for all } f \in L_{\alpha}^{p} .
$$

In particular, if the Fourier transform of $f$ is supported in $A$, then $\|f\|_{\Lambda_{\beta}} \leqslant c\|f\|_{I_{. k}^{\prime \prime}}$.

Proof. By Theorem 2.1, it suffices to establish the result for $p=2$. Now $f \in L_{\alpha}^{2}$ means $F=J_{\alpha} g$ for $g \in L^{2}$. Thus by (2.7) it suffices to bound uniformly $\left\|\tau * P_{A} J_{\alpha} g\right\|_{\infty}$ and $2^{k \beta}\left\|\sigma_{k} * P_{A} J_{\alpha} g\right\|_{\infty}$, where $\beta=\alpha-d / 2$. The first is trivial; for the second we use $\sigma_{k} * P_{A} J_{\alpha} g=m_{k} * g$, where $\hat{m}_{k}(\xi)=\hat{\sigma}_{k}(\xi) \chi_{A}(\xi)\left(1+|\xi|^{2}\right)^{-d / 2}$. Since

$$
\left\|m_{k} * g\right\|_{x} \leqslant\left\|m_{k}\right\|_{2}\|g\|_{2}=\left\|\hat{m}_{k}\right\|_{2}\|g\|_{2} \text {, }
$$

and from (2.1) we obtain

$$
\begin{aligned}
\left\|\hat{m}_{k}\right\|_{2}^{2} & \leqslant c \int_{2^{k-1} \leqslant|\xi| \leqslant 2^{k+1}}|\xi|^{-2 \alpha} \chi_{A}(\xi) d \xi \\
& \leqslant c 2^{-2 k \alpha}\left|\left\{\xi \in A:|\xi| \leqslant 2^{k+1}\right\}\right| \leqslant c 2^{(d-2 \alpha) k},
\end{aligned}
$$

we can conclude that $2^{k \beta}\left\|\sigma_{k} * P_{A} J_{\alpha} g\right\|_{\infty}$ is uniformly bounded. Q.E.D.

THEOREM 2.4. Fix $A$ of asymptotic dimension $\leqslant d$, and let $\alpha=d / p$ with $1<p \leqslant 2$. Then there exists a constant $\lambda_{0}$, depending only on $p$ and the constant in (2.1), such that if $\hat{f}$ is supported in $A, f \in L_{\alpha}^{p}$ and $\lambda\|f\|_{L_{\alpha}^{p}}^{p^{\prime}}<\lambda_{0}$, then $\exp \left(\lambda|f|^{p^{\prime}}\right)-1$ is integrable.

Proof. It suffices to establish the estimate

$$
\|f\|_{q}^{q} \leqslant c^{q}\left(1+q / p^{\prime}\right)^{1+q / p^{\prime}}\|f\|_{L_{\alpha}^{p}}^{q}
$$

for all $q \geqslant p$, for then the result follows by summing the power series expansion of exp as in [13]. Since $f \in L^{p}$ it suffices to establish (2.9) for $q$ sufficiently large. We will give a separate proof for $1<p<2$ and $p=2$.

Suppose $1<p<2$. Define $h$ by $\hat{h}(\xi)=\chi_{A}(\xi)\left(1+|\xi|^{2}\right)^{-\alpha / 2}$. Then there exists $g \in L^{p}$ with $\|f\|_{L_{\alpha}^{p}}=\|g\|_{p}$ such that $\hat{f}(\xi)=\chi_{A}(\xi)\left(1+|\xi|^{2}\right)^{-\alpha / 2} \hat{g}(\xi)$, so $f=h * g$. Thus to establish (2.9) we need the estimates

$$
\|h\|_{s} \leqslant c\left(1+q / p^{\prime}\right)^{1 / q+1 / p^{\prime}}
$$

where $1 / s=1 / q+1 / p^{\prime}$. Note that for $q$ large, $s$ is close to $p^{\prime}$, so $s / 2-1$ is bounded away from zero (here we use $p<2$ ), but $s<p^{\prime}$. Now from (2.1) we have $\hat{h} \in L^{p, \infty}$, and since $\hat{h} \in L^{\infty}$ we also have $\hat{h} \in L^{2}$. Thus $h \in L^{p^{\prime}, \infty} \cap L^{2}$, and we 
claim this implies (2.10). Indeed

$$
\begin{aligned}
\|h\|_{s}^{s} & =\int_{0}^{\infty} h^{*}(t)^{s} d t \leqslant c \int_{0}^{1} t^{-s / p^{\prime}} d t+c \int_{1}^{\infty} t^{-s / 2} d t \\
& =c\left(1-s / p^{\prime}\right)^{-1}+c(s / 2-1)^{-1} \leqslant c^{\prime}\left(1+q / p^{\prime}\right) .
\end{aligned}
$$

Next let $p=2$. We will establish (2.9) via the Hausdorff-Young inequality and estimates for $\|\hat{f}\|_{q^{\prime}}$. Now

$$
\|\hat{f}\|_{q^{\prime}}=\|\hat{h} \hat{g}\|_{q^{\prime}} \leqslant\|\hat{h}\|_{r}\|\hat{g}\|_{2}
$$

where $1 / r=1 / 2-1 / q$, so it suffices to show

$$
\|\hat{h}\|_{r} \leqslant c(1+q / 2)^{1 / q+1 / 2} \text {. }
$$

But

$$
\begin{aligned}
\|\hat{h}\|_{r}^{r} & =\int_{A}\left(1+|\xi|^{2}\right)^{-r d / 4} d \xi=\int_{0}^{\infty}\left(1+t^{2}\right)^{-r d / 4} d|\{\xi \in A:|\xi| \leqslant t\}| \\
& \leqslant \frac{r d}{2} \int_{0}^{\infty} t\left(1+t^{2}\right)^{-1-r d / 4} c t^{d} d t \leqslant c\left(1+\frac{q}{2}\right) . \quad \text { Q.E.D. }
\end{aligned}
$$

REMARKS. The method of proof for $p=2$ does not give the optimal result for $p<2$. The embedding of $L_{\alpha}^{p}$ in BMO is proved in Stein and Zygmund [9] when $\alpha=n / p$. It seems unlikely that the analogous result holds here.

Next we consider improvements in the Sobolev inequalities concerning restrictions to subspaces. Let $V$ be any affine subspace of $\mathbf{R}^{n}$ of dimension $k$ and codimension $m=n-k$. Let $R_{V}$ denote the operator of restriction to $V$ which is not a priori defined for functions which are not continuous. Nevertheless $R_{V}$ is well defined on $L_{\alpha}^{p}\left(\mathbf{R}^{n}\right)$ if $\alpha>m / p$, and the Sobolev inequalities are

$$
\left\|R_{V} f\right\|_{L_{\beta}^{q}} \leqslant c\|f\|_{L_{\alpha}^{p}}
$$

provided $1<p<q<\infty, \alpha>m / p$, and

$$
1 / p-1 / q=(1 / k)(\alpha-\beta-m / p)
$$

(or equivalently $\alpha-\beta=n / p-k / q$ ). If we want $p=q$ we must have $p<2$ (for a more precise result involving Besov spaces see $[7,16,17])$. The identity $(2.12)$ should be thought of as follows: $m / p$ derivatives are spent in restricting to $V$, after which the rate of exchange of derivatives for increased integrability is the same as on a $k$-dimensional space.

Now suppose $A$ has asymptotic dimension $\leqslant d$ and $\hat{f}$ has support in $A$. We can first apply Theorem 2.1 to get $f \in L_{\beta_{1}}^{q_{1}}$ (provided $p \leqslant 2 \leqslant q_{1}$ ) and then (2.11). If $d \leqslant k$ then it is worthwhile to take $q_{1}$ as large as possible. We then find that (2.12) can be replaced by

$$
1 / p-1 / q=(1 / d)(\alpha-\beta-m / q)-\varepsilon
$$

for $\varepsilon>0$, but we can take $\varepsilon=0$ only in the case $q=2$ when the choice $q_{1}=q$ is allowed in (2.11). In place of $\alpha>m / p$ we now require

$$
\alpha>d / p+(m-d) / 2,
$$

which is a weaker condition if $d<m$, and a stronger condition if $d<m$. 
For some sets $A$ it is possible to improve on (2.13) and (2.14). We begin with a simple result where the condition on $A$ is given in a rather cumbersome form.

Lemma 2.5. Fix $A$ and suppose

$$
h\left(\xi^{\prime}\right)=\left(1+\left|\xi^{\prime}\right|^{2}\right)^{\beta / 2}\left(\int_{\mathbf{R}^{m}} \chi_{A}(\xi)\left(1+|\xi|^{2}\right)^{-\alpha p / 2} d \xi_{1} \cdots d \xi_{m}\right)^{1 / p}
$$

belongs to $L^{r, \infty}\left(\mathbf{R}^{k}\right)$, where $\xi^{\prime}=\left(\xi_{m+1}, \ldots, \xi_{n}\right)$. Then (2.11) holds for all $f \in L_{\alpha}^{p}$ with $\hat{f}$ supported in $A$, where $V=\left\{x: x_{1}=0, \ldots, x_{m}=0\right\}$, provided $1<p \leqslant 2 \leqslant q<\infty$, $p^{\prime} \leqslant q$ and $1 / p-1 / q=1 / r$.

Proof. We have $\hat{f}(\xi)=\chi_{A}(\xi)\left(1+|\xi|^{2}\right)^{-\alpha / 2} \hat{g}(\xi)$ for some $g \in L^{p}$ with $\|g\|_{p}=$ $\|f\|_{L_{\alpha}^{p}}$, and

$$
\left(R_{V} f\right)^{\hat{n}}\left(\xi_{m+1}, \ldots, \xi_{n}\right)=\int_{\mathbf{R}^{m}} \chi_{A}(\xi)\left(1+|\xi|^{2}\right)^{-\alpha / 2} \hat{g}(\xi) d \xi_{1} \cdots d \xi_{m} .
$$

Since $p \leqslant 2$ we have $\hat{g} \in L^{p^{\prime}}\left(\mathbf{R}^{n}\right)$, and since $q \geqslant 2$ it suffices to show

$$
\left(1+\left|\xi^{\prime}\right|^{2}\right)^{\beta / 2}\left(R_{V} f\right)^{\hat{\prime}}\left(\xi^{\prime}\right) \in L^{q^{\prime}, q}\left(\mathbf{R}^{k}\right) .
$$

Thus it suffices to show that the operator

$$
T \phi\left(\xi^{\prime}\right)=\left(1+\left|\xi^{\prime}\right|^{2}\right)^{\beta / 2} \int_{\mathbf{R}^{m}} \chi_{A}(\xi)\left(1+|\xi|^{2}\right)^{-\alpha / 2} \phi(\xi) d \xi_{1} \cdots d \xi_{m}
$$

is bounded from $L^{p^{\prime}}\left(\mathbf{R}^{n}\right)$ to $L^{q^{\prime}, q}\left(\mathbf{R}^{k}\right)$. But by Hölder's inequality we have

$$
\left|T \phi\left(\xi^{\prime}\right)\right| \leqslant h\left(\xi^{\prime}\right)\left(\int|\phi(\xi)|^{p^{\prime}} d \xi_{1} \cdots d \xi_{m}\right)^{1 / p^{\prime}}
$$

and multiplication by a function in $L^{r \cdot x}$ is a bounded operator from $L^{p^{\prime}}$ to $L^{q^{\prime} \cdot q}$ provided $p^{\prime} \leqslant q$. Q.E.D.

Now let $V$ be an arbitrary $k$-dimensional affine subspace. Let us say that $A$ is thin perpendicular to $V$ if there exists a constant $M$ such that the $m$-dimensional measure of $A$ intersected with every $m$-dimensional affine subspace perpendicular to $V$ is at most $M$. If $V$ is $\left\{x: x_{1}=0, \ldots, x_{m}=0\right\}$ the condition is $\int \chi_{A}(\xi) d \xi_{1} \cdots d \xi_{m} \leqslant M$ for all $\xi^{\prime}$. This condition clearly implies that $A$ has asymptotic dimension $\leqslant k$. The next theorem shows that we can have (2.11) for the same value of $q$ as in Theorem 2.1.

THEOREM 2.6. Fix a set $A$ which is thin perpendicular to $V$. Then (2.11) holds for all $f \in L_{\alpha}^{p}$ with $\hat{f}$ supported in $A$, provided $\alpha>0,1<p \leqslant 2 \leqslant q<\infty$ and $1 / p-1 / q=$ $(\alpha-\beta) / k$.

PROOF. Without loss of generality we may take $V=\left\{x: x_{1}=0, \ldots, x_{m}=0\right\}$ as in Lemma 2.5. We then have the estimate

$$
h\left(\xi^{\prime}\right) \leqslant M^{1 / p}\left(1+\left|\xi^{\prime}\right|^{2}\right)^{(\beta-\alpha) / 2}
$$

since $\alpha>0$, and so $h \in L^{r, \infty}$ if $1 / r=(\alpha-\beta) / k$. The condition $p^{\prime} \leqslant q$ in the lemma can be removed as follows. The operator $T$ in the proof of the lemma is 
bounded from $L^{p^{\prime}}\left(\mathbf{R}^{n}\right)$ to $L^{q^{\prime} \cdot q}\left(\mathbf{R}^{k}\right)$, where now $p$ and $q$ can vary, with $1 / p-1 / q=$ $(\alpha-\beta) / k$. Hence it follows by the real interpolation method that it is also bounded from $L^{p^{\prime} \cdot p}\left(\mathbf{R}^{n}\right)$ to $L^{q^{\prime} \cdot q}\left(\mathbf{R}^{k}\right)$ since $p \leqslant q$, and this suffices to complete the proof since $\hat{g} \in L^{p^{\prime} \cdot p}\left(\mathbf{R}^{n}\right)$. Q.E.D.

3. Compact symmetric spaces. Let $X$ be either a compact Lie group $G$ or a compact symmetric space $G / K$ with invariant measure $d \mu$ normalized to have total mass one. Let $L^{-}$index by highest weight $m$ the irreducible representations $\pi_{m}$ of $G$ that occur in $L^{2}(X)$, let $E_{m}$ denote the subspace of $L^{2}(X)$ of all functions transforming according to the representation $\pi_{m}$, and let $d_{m}$ denote the dimension of $E_{m}$ (this is the dimension of $\pi_{m}$ if $X=G / K$ and the square of the dimension of $\pi_{m}$ if $X=G$ ). Choose an orthonormal basis $\phi_{m k}, k=1, \ldots, d_{m}$, for $E_{m}$ and let

$$
Z_{m}(x, y)=\sum_{k} \phi_{m k}(x) \overline{\phi_{m k}(y)}
$$

be the reproducing kernel for $E_{m}, f(x)=\int Z_{m}(x, y) f(y) d \mu(y)$ for $f \in E_{m} . Z_{m}$ is independent of the choice of basis, so $Z_{m}(x, x)$ is a constant independent of $x$. Since $\int Z_{m}(x, x) d \mu(x)=d_{m}$, we deduce $Z_{m}(x, x)=d_{m}$ and, from this, the basic estimate

$$
\|f\|_{\infty} \leqslant d_{m}^{1 / 2}\|f\|_{2} \text { for } f \in E_{m} .
$$

Now for $f \in L^{2}(X)$ we consider the Fourier series expansion

$$
f=\sum_{m \in \mathfrak{L}} d_{m}^{1 / 2} f_{m}
$$

where

$$
f_{m}=d_{m}^{-1 / 2} \int Z_{m}(x, y) f(y) d \mu(y)
$$

is the component of $f$ in $E_{m}$ (the factors $d_{m}^{ \pm 1 / 2}$ are chosen to make the estimates below come out neatly). Let $d \nu$ denote the discrete measure on $\mathcal{L}$ which assigns to $m$ the weight $d_{m}$. Then we have the estimates

$$
\begin{aligned}
& \|f\|_{I_{L^{2}(d \mu)}}=\left\|\left\{\left\|f_{m}\right\|_{2}\right\}\right\|_{L^{2}(d \nu)}, \\
& \|f\|_{I^{\infty}(d \mu)} \leqslant\left\|\left\{\left\|f_{m}\right\|_{2}\right\}\right\|_{L^{\prime}(d \nu)},
\end{aligned}
$$

and

$$
\left\|\left\{\left\|f_{m}\right\|_{2}\right\}\right\|_{L^{\infty}(d \nu)} \leqslant\|f\|_{L^{\prime}(d \mu)} .
$$

Indeed (3.4) is just the Parseval identity, (3.5) follows from (3.2) and (3.1), and (3.6) follows from

$$
\left\|f_{m}\right\|_{2}^{2}=d_{m}^{-1 / 2} \int f_{m}(x) \overline{f(x)} d \mu(x) \leqslant d_{m}^{-1 / 2}\left\|f_{m}\right\|_{\infty}\|f\|_{1}
$$

and (3.1). By interpolating these estimates we obtain the analogues of the Hardy-Littlewood inequalities ((2.5) and (2.6)):

$$
\left\|\left\{\left\|f_{m}\right\|_{2}\right\}\right\|_{L^{p^{\prime}, p}(d \nu)} \leqslant\|f\|_{L^{p}(d \mu)} \text { if } 1<p<2,
$$

and

$$
\|f\|_{L^{q}(d \mu)} \leqslant c\left\|\left\{\left\|f_{m}\right\|_{2}\right\}\right\|_{L^{q^{\prime} \cdot q}} \text { if } 2<q<\infty .
$$


Next we consider Sobolev spaces on $X$. The set $E$ of highest weights is naturally identified with a subset of $\mathbf{Z}^{r}$, the dual group to a maximal torus in $G$. The Laplacian $\Delta$ on $G$ is diagonalized by the Fourier series (3.2). We have

$$
-\Delta f_{m}=\left(|m+\rho|^{2}-|\rho|^{2}\right) f_{m}
$$

where $\rho$ is one-half the sum of the positive roots. Thus $|\rho|^{2} I-\Delta$ is an invertible second-order elliptic differential operator, and its powers

$$
\left(|\rho|^{2} I-\Delta\right)^{z / 2} \sum d_{m}^{1 / 2} f_{m}=\sum|m+\rho|^{z} d_{m}^{1 / 2} f_{m}
$$

serve to define the Sobolev spaces

$$
L_{\alpha}^{p}(X)=\left\{f:\left(|\rho|^{2} I-\Delta\right)^{\alpha / 2} f \in L^{p}\right\}=\left\{\left(|\rho|^{2} I-\Delta\right)^{-\alpha / 2} g: g \in L^{p}\right\} .
$$

Since $X$ is compact this definition is equivalent to any other reasonable definition.

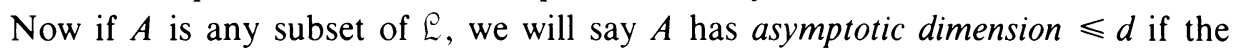
inequality

$$
\nu(\{m \in A:|m| \leqslant s\})=\sum_{\substack{m \in A \\|m| \leqslant s}} d_{m} \leqslant c s^{d}
$$

holds for some constant $c$ and all $s>1$. The fact that the full set $\mathcal{L}$ has asymptotic dimension exactly equal to $\operatorname{dim} X$ is essentially equivalent to Weyl's theorem that the asymptotic behavior of the number of eigenfunctions of $-\Delta$ with eigenvalue $\leqslant t^{2}$ is exactly $O\left(t^{\operatorname{dim} X}\right)$. An alternate proof of this fact for the case $X=G$ can be given using a different theorem of Weyl-the dimension formula for $d_{m}$.

Now we have all the ingredients for proving improved Sobolev inequalities for functions with Fourier series $f=\sum_{m \in A} d_{m}^{1 / 2} f_{m}$. Let $P_{A}$ denote the orthogonal projection

$$
P_{A}\left(\sum_{m \in \mathcal{L}} d_{m}^{1 / 2} f_{m}\right)=\sum_{m \in A} d_{m}^{1 / 2} f_{m} .
$$

TheOREM 3.1. Fix $A$ of asymptotic dimension $\leqslant d$. Then

$$
\left\|P_{A} f\right\|_{L_{\beta}^{q}} \leqslant c\|f\|_{L_{\alpha}^{p}} \text { for all } f \in L_{\alpha}^{p}
$$

provided $1<p \leqslant 2 \leqslant q<\infty$ and $1 / p-1 / q=(\alpha-\beta) / d$.

REMARKS. We omit the proof, since it is essentially the same as the proof of Theorem 2.1. The analogues of Theorems 2.2-2.4 also hold; again the proofs are essentially the same, except that for the embedding into Lipschitz-Zygmund spaces the analogue of the characterization (2.7) is not available (although it is very likely to be true). Instead we may use the sufficient condition

$$
\sum_{\lambda \leqslant|m| \leqslant 2 \lambda} d_{m}\left\|f_{m}\right\|_{2} \leqslant M \lambda^{-\alpha} \text { for all } \lambda>0
$$

implies $f \in \Lambda_{\alpha}$. A simple proof, communicated by David Ragozin, is as follows: from (3.11) and (3.1) we obtain

$$
\left\|f-\sum_{|m| \leqslant \lambda} d_{m}^{1 / 2} f_{m}\right\|_{\infty} \leqslant M \lambda^{-\alpha},
$$

and this implies $f \in \Lambda_{\alpha}$ by generalizations of Bernstein's inequalities $[\mathbf{5}, \mathbf{6}]$. 
To prove the analogue of Theorem 2.3 we argue that if $f \in L_{\alpha}^{2}$ then

$$
\sum_{\substack{\lambda \leqslant|m| \leqslant 2 \lambda \\ m \in A}} d_{m}\left\|f_{m}\right\|_{2} \leqslant\left(\sum|m+\rho|^{2 \alpha} d_{m}\left\|f_{m}\right\|_{2}^{2}\right)^{1 / 2}\left(\sum_{\substack{\lambda \leqslant|m| \leqslant 2 \lambda \\ m \in A}} d_{m}|m+\rho|^{-2 \alpha}\right)^{1 / 2} .
$$

The first factor on the right side is just $\|f\|_{L_{\alpha}^{2}}$, while the second can be estimated (using $|m+\rho|^{-2 \alpha} \leqslant c \lambda^{-2 \alpha}$ since $|m| \geqslant \lambda$ ) by $c \lambda^{-\alpha}\left(\sum_{|m| \leqslant 2 \lambda: m \in A} d_{m}\right)^{1 / 2}$, so $P_{A} f \in \Lambda_{\beta}$ by (3.11) if $\beta=\alpha-d / 2$ if $A$ has asymptotic dimension $\leqslant d$.

4. Radon transforms on spheres. Suppose $X_{1}=G / K_{1}$ and $X_{2}=G / K_{2}$ are two compact symmetric spaces with the same isometry group $G$, with $\operatorname{dim} X_{1}=n_{1}<$ $\operatorname{dim} X_{2}=n_{2}$. If $T: L^{2}\left(X_{1}\right) \rightarrow L^{2}\left(X_{2}\right)$ commutes with the action of $G$, then the results of $\$ 3$ show that on the range of $T$ we may use the dimension $n_{1}$ rather than $n_{2}$ in the Sobolev inequalities.

Let $E_{1}$ and $E_{2}$ denote the lattices of highest weights $m$ of representations $\pi_{m}$ of $G$ appearing in $L^{2}\left(X_{1}\right)$ and $L^{2}\left(X_{2}\right)$, respectively, and let $E=E_{1} \cap \mathcal{L}_{2}$. Then by Schur's lemma the operator $T$ must have the form

$$
T\left(\sum_{m \in E_{1}} d_{m}^{1 / 2} f_{m}\right)=\sum_{m \in E^{\prime}} \gamma_{m} d_{m}^{1 / 2} f_{m}^{\prime}
$$

for some constants $\gamma_{m}$, where the mapping $f_{m} \rightarrow f_{m}^{\prime}$ is an isometry (unique up to scalar factor of absolute value one) intertwining the representation $\pi_{m}$ as realized (with multiplicity one) on $X_{1}$ and $X_{2}$.

THEOREM 4.1. Suppose we have $\left|\gamma_{m}\right| \leqslant c|m|^{-\alpha}$ for all $m \in \mathcal{L}$. Then $T$ is a bounded operator from $L^{2}\left(X_{1}\right)$ to $L^{q}\left(X_{2}\right)$ provided $0<\alpha<n_{1} / 2$ and $1 / 2-1 / q=\alpha / n_{1}$. If $\alpha>n_{1} / 2$ then $T$ is a bounded operator from $L^{2}\left(X_{1}\right)$ to $\Lambda_{\beta}\left(X_{2}\right)$ where $\beta=\alpha-n_{1} / 2$.

Proof. From the hypotheses $T$ is a bounded operator from $L^{2}\left(X_{1}\right)$ to $L_{\alpha}^{2}\left(X_{2}\right)$.

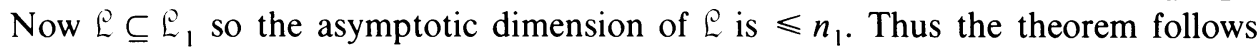
from Theorem 3.1 and the remarks following. Q.E.D.

Let $G_{n, k}$ denote the Grassmannian manifold of $k$-dimensional subspaces of $\mathbf{R}^{n}$. Then $G_{n, k}$ may be identified with the symmetric space $O(n) / O(n-k) \times O(k)$ and has dimension $(n-k) k$. The $k$-dimensional Radon transform $L_{k}$ on the $n$-dimensional sphere $S^{n}$ (or the projective space $X$ obtained by identifying antipodal points, $\left.X=G_{n+1,1}\right)$ can be regarded as an operator from $L^{2}(X)$ to $L^{2}\left(G_{n+1, k+1}\right)$, which commutes with the action of $G=O(n+1)$. In [14] we computed the constants $\gamma_{m}$ for $L_{k}$ and found the estimate $\left|\gamma_{m}\right| \leqslant c|m|^{-k / 2}$. Thus Theorem 4.1 shows that $L_{k}$ is bounded from $L^{2}(X)$ to $L^{q}\left(G_{n+1, k+1}\right)$ where $1 / q=1 / 2-k / 2 n$. Combining this with other results from [14, Corollary 4.6] we obtain

THEOREM 4.2. $L_{k}$ is bounded from $L^{p}(X)$ to $L^{q}\left(G_{n+1, n+k}\right)$ provided the point $\left(\frac{1}{p}, \frac{1}{q}\right)$ lies in the convex hull of the points $(0,0),(1,1),\left(\frac{1}{2}, \frac{1}{2}-\frac{k}{2 n}\right),\left(\frac{1}{2}+\frac{k}{2 n}, \frac{1}{2}\right)$.

5. Solutions of the wave equations. Let $u(x, t)$ for $x \in \mathbf{R}^{n}$ and $t \in \mathbf{R}$ be a solution of the wave equation $u_{t t}=\Delta_{x} u$. Then the Fourier transform of $u$ in all $n+1$ variables is supported in the cone $\tau^{2}=|\xi|^{2}$, and if we convolve $\hat{u}$ with a function $\phi$ of compact support (hence multiply $u$ by $\mathscr{F}^{-1}(\phi)$ ), we will obtain a function 
supported in a neighborhood of the cone. Since a neighborhood of the cone has asymptotic dimension $\leqslant n$, we can expect that $u$ will satisfy improved Sobolev inequalities with $d=n$ locally. We cannot expect $u$ to satisfy global Sobolev inequalities since a function with Fourier transform supported on the cone cannot be in $L^{p}$ for $p \leqslant 2$.

We will see in this section that these expectations are borne out, but more interestingly we will see that the situation is much more complicated. For some values of $p$ we can obtain local improved Sobolev inequalities with $d$ as low as $(n+1) / 2$, while for other values of $p(>2$, of course), the usual Sobolev inequalities (with $d=n+1$ ) cannot be improved. These counterexamples show that the restriction $p \leqslant 2 \leqslant q$ in Theorem 2.1 cannot be entirely dispensed with.

To simplify the discussion we will consider the case of one derivative only. Thus let $\nabla u$ belong to $L^{p}$ locally (in space-time) for $u$ a solution of the wave equation. When does this imply that $u$ belongs to $L^{q}$ locally? The usual Sobolev inequalities give the embedding for $1 / p-1 / q=1 /(n+1)$, so the question really is whether we can do better. For the affirmative result we need only reinterpret our results in [10].

THEOREM 5.1. Let $\nabla$ u belong to $L^{p}$ in every bounded subset of $\mathbf{R}^{n+1}, n \geqslant 2$, and let $u$ satisfy the wave equation. Then $u$ is in $L^{q}$ in every bounded subset of $\mathbf{R}^{n+1}$, provided

$$
2\left(\frac{n+1}{n+3}\right) \leqslant p \leqslant 2 \text { and } \frac{n}{q}=\frac{1}{p}+\frac{n-3}{2},
$$

or

$$
\frac{2 n}{n+2} \leqslant p \leqslant 2\left(\frac{n+1}{n+3}\right) \text { and } \frac{1}{q}=\frac{n}{p}-\frac{n+1}{2}
$$

(also $p>1$ if $n=2$ ). If $K$ is any bounded subset of $\mathbf{R}^{n+1}$ there exists a bounded neighborhood $K^{\prime}$ of $K$ such that

$$
\left(\int_{K}|u(x, t)|^{q} d x d t\right)^{1 / q} \leqslant c\left(\int_{K^{\prime}}|\nabla u(x, t)|^{p} d x d t\right)^{1 / p}
$$

with the constant $c$ depending only on the diameter of $K$ in the t-variable.

PROOF. In [10, Theorem 2] we proved

$$
\left(\int_{\mathbf{R}^{n}}|u(x, t+s)|^{q} d x\right)^{1 / q} \leqslant c(t)\left(\int_{\mathbf{R}^{n}}|\nabla u(x, s)|^{p} d x\right)^{1 / p}
$$

for solutions of the wave equation, under the stated conditions on $p$ and $q$, where the constant $c(t)$ is finite for every $t \neq 0$. In fact it is clear from the proof that $c(t)$ is uniformly bounded on compact intervals not containing the origin, and in [11] we observed that a simple homogeneity argument shows $c(t)=c t^{-\alpha}$ for $\alpha=n / p-n / q$ -1 .

Now given $K$ we can enlarge it if necessary so that it is a product of a bounded set $K_{0}$ in $\mathbf{R}^{n}$ and an interval $[a, b]$ in $\mathbf{R}$. We then choose $K^{\prime}=\left\{(x, t): x \in K_{0}^{\prime}\right.$ and $a-1 \leqslant t \leqslant b+1\}$, where $K_{0}^{\prime}$ is chosen so that that the Cauchy data at $t=t_{0}$ in $(a-1, b+1)$ and $x \in K_{0}^{\prime}$ for a solution of the wave equation determine the 
solution in all of $K$. Since $\nabla u \in L^{p}\left(K^{\prime}\right)$, it follows by Fubini's theorem that the Cauchy date of $u$ for $t=t_{0}$ in $[a-1, b+1]$ is in $L^{p}\left(K_{0}^{\prime}\right)$ for almost every $t_{0}$. Fixing such a $t_{0}$, let $v$ be the solution to the wave equation in $\mathbf{R}^{n+1}$ determined by the Cauchy data of $u$ at $t=t_{0}$ extended to be zero for $x$ outside $K_{0}^{\prime}$. Then $v=u$ in $K$ and

$$
\int_{\mathbf{R}^{n}}\left|\nabla v\left(x, t_{0}\right)\right|^{p} d x=\int_{K_{0}^{\prime}}\left|\nabla u\left(x, t_{0}\right)\right|^{p} d x
$$

Thus (5.2) gives

$$
\left(\int_{K_{0}}\left|u\left(x, t+t_{0}\right)\right|^{q} d x\right)^{1 / q} \leqslant c(t)\left(\int_{K_{0}^{\prime}}\left|\nabla u\left(x, t_{0}\right)\right|^{p} d x\right)^{1 / p} .
$$

From this it is trivial to get $(5.1)$ by letting $t_{0}$ vary discretely and integrating with respect to the $t$ variable over $1 / 2 \leqslant|t| \leqslant 1$. Q.E.D.

Remarks. (1) At the endpoints $p=2$ and $p=2 n /(n+2)$ (hence $q=2$ ), the value of $d=\left(\frac{1}{p}-\frac{1}{q}\right)^{-1}$ is exactly $n$ as expected, but elsewhere it is smaller, achieving the minimum value $(n+1) / 2$ at $p=2((n+1) /(n+3)), q=2((n+1) /(n-1))$.

(2) Note that the proof actually establishes the stronger conclusion

$$
\sup _{a \leqslant t \leqslant b}\left(\int_{K_{0}}|u(x, t)|^{q} d x\right)^{1 / q} \leqslant c\left(\int_{K^{\prime}}|\nabla u(x, t)|^{p} d x d t\right)^{1 / p} .
$$

This is not surprising in view of Theorem 2.6.

(3) Brenner [1] has extended the results of [10] to a large class of hyperbolic partial differential operators, so the theorem continues to hold for solutions of these equations.

We turn now to some simple counterexamples. Let $n=3$; this choice is particularly convenient since any function of the form $u(x, t)=(\phi(r+t)-\phi(r-t)) / r$, where $r=|x|$ and $\phi$ is a locally integrable function of one variable, is a solution to the wave equation. If we choose $\phi(r)=|r|^{\lambda}$ for $r$ near 0 with $0<\lambda<1$, such that $\phi$ is $C^{\infty}$ and of compact support in $r>0$, then $u$ cannot belong to $L^{q}$ near the origin if $q \geqslant 4 /(1-\lambda)$. On the other hand an easy computation shows that $\nabla u$ is locally in $L^{p}$ provided $p<4 /(2-\lambda)$ and $p<1 /(1-\lambda)$. Now for $p$ in the interval $3 \leqslant p<4$, we can choose $\lambda=2-4 / p+\varepsilon$ and $\nabla u$ will be in $L^{p}$ locally, while $u$ will not be in $L^{q}$ locally unless $1 / p-1 / q \leqslant 1 / 4$, so there can be no improvement on the usual Sobolev inequality.

By multiplying the above example by a fixed function $g(x, t)$ such that $\hat{g}$ is in $\mathcal{D}$, we obtain a global counterexample to extending Theorem 2.1 into the range $q>p \geqslant 3$, at least for $n=4, \alpha=1$, and $A$ a neighborhood of the cone (with asymptotic dimension 3).

6. Noncompact symmetric spaces. Let $X$ be a noncompact symmetric space, $X=G / K$ where $G$ is a semisimple Lie group and $K$ a maximal compact subgroup. Then there is a Fourier expansion

$$
f=w^{-1} \int_{\mathbf{R}^{\nu}} \phi_{\lambda} * f|\mathbf{c}(\lambda)|^{-2} d \lambda
$$


for functions $f \in L^{2}(X)$, where $\phi_{\lambda}$ are the spherical functions, $w$ is the order of the Weyl group, $\mathbf{c}(\lambda)$ is the Harish-Chandra c-function, and $\nu$ is the rank of $G$ (the parameter space $\mathbf{R}^{\nu}$ is canonically identified with the dual space of $\mathfrak{A}$, the abelian component in the Iwasawa decomposition of (S). If desired one can restrict the $\lambda$ parameter to a Weyl chamber, or equivalently require every function of $\lambda$ to be invariant under the Weyl group. See Helgason [4] for details.

Because of the Kunze-Stein phenomena [3], the Fourier expansion of a function in $L^{p}$ for $p<2$ will be analytic in $\lambda$, so there can be no such (nonzero) functions whose Fourier expansions are supported on nontrivial subsets. Nevertheless, we can obtain meaningful improved Sobolev inequalities by considering the $L^{2}$ orthogonal projection $P_{A}$ onto the portion of the Fourier expansion lying in $A$. Here $A$ is any measurable set of nonzero measure that is Weyl invariant, and

$$
P_{A} f=w^{-1} \int_{A} \phi_{\lambda} * f|\mathbf{c}(\lambda)|^{-2} d \lambda .
$$

The Sobolev spaces $L_{\alpha}^{p}(X)$ can be defined in terms of the Laplacian $\Delta$ and the pseudodifferential operators $(-\Delta)^{-\alpha / 2}$, which are most simply expressed in terms of the Fourier expansion

$$
(-\Delta)^{-\alpha / 2} f=w^{-1} \int_{\mathbf{R}^{\nu}}\left(|\rho|^{2}+|\lambda|^{2}\right)^{-\alpha / 2} \phi_{\lambda} * f|\mathbf{c}(\lambda)|^{-2} d \lambda,
$$

where $\rho$ denotes half the sum of the positive roots (it is the appearance of the positive constant $|\rho|^{2}$ in (6.3) that makes it superfluous to add a constant to $-\Delta$ as in the Euclidean or compact cases). We take $L_{\alpha}^{p}$ to be the image of $L^{p}$ under $(-\Delta)^{-\alpha / 2}$, $f \in L_{\alpha}^{p}$ if and only if there exists $g \in L^{p}$ with $f=(-\Delta)^{-\alpha / 2} g$. It is not hard to show (by slight modifications of the arguments of [12]) that this definition is equivalent with all other reasonable definitions (there is a unique space invariant under the group action and, for functions supported in a compact coordinate patch, equivalent to the usual Sobolev space), and the global Sobolev inequalities $\|f\|_{L_{\beta}^{q}} \leqslant c\|f\|_{L_{\alpha}^{p}}$ hold if $1<p \leqslant q<\infty$ and $1 / p-1 / q=(\alpha-\beta) / n$, where $n$ denotes the dimension of $X$.

The improved Sobolev inequalities we seek are of the form $\left\|P_{A} f\right\|_{L_{\beta}^{q}} \leqslant c\|f\|_{L_{\alpha}^{p}}$, where $1 / p-1 / q=(\alpha-\beta) / d$ and $1<p \leqslant 2 \leqslant q<\infty$.

Definition 6.1. A Weyl group invariant subset $A \subseteq \mathbf{R}^{\nu}$ is said to have asymptotic dimension $\leqslant d$ if the estimate

$$
\int_{A \cap\{|\lambda| \leqslant s\}}|\mathbf{c}(\lambda)|^{-2} d \lambda \leqslant c s^{d}
$$

holds for all $s>1$ and some constant $c$.

LEMMA 6.2. The asymptotic dimension of $\mathbf{R}^{\nu}$ is equal to $n=\operatorname{dim} X$.

Proof. By a theorem of Gindikin and Karpelevic (see [4]) we have

$$
\mathbf{c}(\lambda)=c \prod_{\alpha \in \Sigma^{+}} \frac{\Gamma\left(\frac{1}{4} m_{2 \alpha}+i \alpha \cdot \lambda /|\alpha|^{2}\right)}{\Gamma\left(\frac{1}{4} m_{2 \alpha}+\frac{1}{2} m_{\alpha}+i \alpha \cdot \lambda /|\alpha|^{2}\right)},
$$


where $\Sigma^{+}$denotes the set of positive roots and $m_{\alpha}$ the multiplicity of the root $\alpha$. Since $n=\nu+\Sigma_{\alpha \in \Sigma^{+}} m_{\alpha}$, the result follows from well-known estimates for the $\Gamma$-function. Q.E.D.

TheOREM 6.3. Let $A \subseteq \mathbf{R}^{\nu}$ have asymptotic dimension $\leqslant d$. Then $\left\|P_{A} f\right\|_{L_{B}^{q}} \leqslant$ $c\|f\|_{L_{\alpha}^{p}}$ provided $1<p \leqslant 2 \leqslant q<\infty$ and $1 / p-1 / q=(\alpha-\beta) / d$.

Proof. Assuming $\beta=0$ for simplicity, we need to show $\left\|P_{A}(-\Delta)^{-\alpha} f\right\|_{q} \leqslant c\|f\|_{p}$. But $P_{A}(-\Delta)^{-\alpha} f=G * f$, where

$$
G=\int_{A}\left(|\lambda|^{2}+|\rho|^{2}\right)^{-\alpha / 2} \phi_{\lambda}|\mathbf{c}(\lambda)|^{-2} d \lambda
$$

Now by interpolating between the Plancherel formula and the uniform boundedness of the spherical functions ( $\left\|\phi_{\lambda}\right\|_{\infty}=1$ in fact), we obtain as before that $G \in L^{r, \infty}$, where $r=d /(d-\alpha)$ provided $d / 2<\alpha<d$. This, together with the weak-type convolutional inequalities, proves the theorem in the case when $1 / p-1 / q>1 / 2$. To eliminate this condition we apply an analytic families interpolation argument, using the obvious $L^{2}$ boundedness of $P_{A}(-\Delta)^{i t}$ for real $t$. Q.E.D.

\section{REFERENCES}

I. P. Brenner, $I_{,},-L_{p}$, estimates for Fourier integral operators related to hyperbolic equations, Math. Z. 145 (1975), $251-254$.

2. R. Coifman and Y. Meyer, Au-delà des opérateurs pseudo-différentiels, Astérisque 57 (1978).

3. M. Cowling, The Kunze-Stein phenomena, Ann. of Math. (2) 107 (1978), 209-234.

4. S. Helgason, Functions on symmetric spaces, Proc. Sympos. Pure Math., vol. 26, Amer. Math. Soc., Providence, R. I., 1973, pp. 101-146.

5. D. Ragozin, Polynomial approximation on compact manifolds and homogeneous spaces, Trans. Amer. Math. Soc. 150 (1970), 41-53.

6. Approximation theory, absolute convergence, and smoothness of random Fourier series on compact Lie groups, Math. Ann. 219 (1976), 1-11.

7. E. M. Stcin, Singular integrals and differentiability properties of functions, Princeton Univ. Press, Princeton, N. J., 1970.

8. E. M. Stcin and (j. Weiss, Introduction to Fourier analysis on Euclidean spaces, Princeton Univ. Press, Princeton, N. J., 1971.

9. E. M. Stein and A. Zygmund, Boundedness of translation invariant operators on Hölder and $L^{p}$ spaces, Ann. of Math. (2) 85 (1967), 337-349.

10. R. Strichartz, Convolutions with kernels having singularities on a sphere, Trans. Amer. Soc. 148 (1970), 461-471.

11. A priori estimates for the wave equation and some applications, J. Funct. Anal. 5 (1970), $218-235$.

12. , Invariant pseudo-differential operators on a Lie group, Ann. Scuola Norm. Sup. Pisa 26 (1972), 587-611.

13. _ A note on Trudinger's extension of Sobolev's inequalities, Indiana Univ. Math. J. 21 (1972), $841-842$.

14. $L^{p}$ estimates for Radon transforms in Euclidean and non-Euclidean spaces, Duke Math. J. 48 (1981), 699-727.

15. G. Warner, Harmonic analysis on semi-simple Lie groups. I, II, Springer, Berlin, 1972.

16. O. V. Besov and S. Nikolskii, Integral representations of functions and imbedding theorems. I, II. Halsted, 1978.

17. S. Nikolskii, Approximation of functions of several variables and imbedding theorems, Springer-Verlag, Berlin and New York, 1975.

Department of Mathematics, Cornell University, Ithaca, New York, 14853 